\title{
Multipolar origin of bound states in the continuum
}

\author{
Zarina Sadrieva, ${ }^{1, *}$ Kristina Frizyuk, ${ }^{1, \dagger}$ Mihail Petrov, ${ }^{1}$ Yuri Kivshar, ${ }^{1,2}$ and Andrey Bogdanov $\circledast^{1}$ \\ ${ }^{1}$ ITMO University, Saint Petersburg 197101, Russia \\ ${ }^{2}$ Nonlinear Physics Center, Australian National University, Canberra ACT 2601, Australia
}

(Received 18 April 2019; revised manuscript received 14 August 2019; published 3 September 2019)

\begin{abstract}
Metasurfaces based on resonant subwavelength photonic structures enable novel methods of wavefront control and light focusing, underpinning a new generation of flat-optics devices. Recently emerged all-dielectric metasurfaces exhibit high-quality resonances underpinned by the physics of bound states in the continuum that drives many interesting concepts in photonics. Here we suggest an approach to explain the physics of bound photonic states embedded in the radiation continuum. We study dielectric metasurfaces composed of planar periodic arrays of Mie-resonant nanoparticles ("meta-atoms") which support both symmetry protected and accidental bound states in the continuum, and employ the multipole decomposition approach to reveal the physical mechanism of the formation of such nonradiating states in terms of multipolar modes generated by isolated meta-atoms. Based on the symmetry of the vector spherical harmonics, we identify the conditions for the existence of bound states in the continuum originating from the symmetries of both the lattice and the unit cell. Using this formalism we predict that metasurfaces with strongly suppressed spatial dispersion can support the bound states in the continuum with the wave vectors forming a line in the reciprocal space. Our results provide a method for designing high-quality resonant photonic systems based on the physics of bound states in the continuum.
\end{abstract}

DOI: 10.1103/PhysRevB.100.115303

\section{INTRODUCTION}

The quest for compact photonic systems with high quality factor $(Q$ factor) modes led to the rapid development of optical bound states in the continuum (BICs). BICs are nonradiating states, characterized by the resonant frequencies embedded to the continuum spectrum of radiating modes of the surrounding space [1-3]. The BICs first appeared as a mathematical curiosity in quantum mechanics [4]. The discovery of BICs in optics immediately attracted broad attention (see, e.g., Refs. [5-7]) due to high potential in applications in communications [8,9], lasing [10-13], filtering [14], and sensing [15-17]. Recent achievements in the field of BICs are discussed in Refs. [18-27].

Decoupling of the resonant mode from the radiative spectrum, which is the basic idea behind the BIC, can be interpreted in several equivalent ways. Within the coupled-mode theory, it corresponds to nullifying the coupling coefficient between the resonant mode and all radiation channels of the surrounding space [28]. Alternatively, the appearance of BICs is explained as vanishing of Fourier coefficients corresponding to open diffraction channels due to the symmetry of the photonic structure. At the particular high-symmetry points of the reciprocal space, for example, at the $\Gamma$ point, the continuous spectrum is divided into the modes of different symmetry classes with respect to the reflectional and rotational symmetry of the photonic system. The bound states of one symmetry class can be found embedded in the continuum

\footnotetext{
*z.sadrieva@metalab.ifmo.ru

${ }^{\dagger}$ k.frizyuk@metalab.ifmo.ru
}

of another symmetry class, and their coupling is forbidden as long as the symmetry is preserved. This kind of BIC is called symmetry protected, and it also allows interpretation in terms of topological charges defined by the number of times the polarization vector winds around the BIC, presented as vortex centers in the polarization field [29]. In contrast to the symmetry protected BIC, the so-called accidental BICs [1,29-32] can be observed out of the $\Gamma$ point due to an accidental nullifying of the Fourier (coupling) coefficients via fine tuning of parameters of the photonic system. Such a mechanism is also known as the Friedrich-Wintgen scenario [33].

There are many methods developed for calculation of transmission/reflection spectra, eigenmodes, and the complex eigenfrequency spectrum of an open periodic system like photonic crystal slabs, metasurfaces, and chains of particles. In particular, these methods include guided mode expansion method [34], resonance state expansion method [35,36], perturbative methods [37,38], Fourier modal method (also known as rigourous coupled wave analysis) [39-41], quasinormal-mode expansion [42,43], coupled Bloch mode approach $[44,45]$, methods using multipole scattering theory [46], and Feshbach projection formalism [47-49]. Many of these methods are used to explain the appearance of BICs in periodic structures and description of their properties [5,29,50-58].

Despite the number of existing approaches to understanding the nature of BICs, there is still room for further development of the theory. During the previous few years the electromagnetic multipole theory [59] has been extensively developed as a natural tool of nanophotonics dealing with the lowest (fundamental) resonances of the system. The main advantage of the multipole decomposition method (MDM) 
is that it provides a representation of an arbitrary field distribution as a superposition of the fields created by a set of multipoles [60,61]. Namely, the multipole expansion has been widely used to determine the polarization and directivity patterns of the scattered field of single particles and their clusters, both plasmonic and dielectric [60-64], for a variety of applications such as polarization control devices [61], dielectric nanoantennas [65], and light demultiplexing [66]. A number of novel optical phenomena have been explained within the MDM such as the anapole effect [67,68], optomechanical phenomena [69,70], and the Kerker effect [71,72].

In this paper, we extend the MDM approach to explain both symmetry protected and accidental BICs. We provide a theory of BIC origin in terms of MDM for a general case of any periodic structure and develop an analytical method, which determines the contribution of the vector spherical harmonics (VSH) to the far field (Sec. II). Working in the VSH basis, we take advantage of the internal symmetry and provide the group-symmetry approach to identifying the BIC formation in terms of the unit-cell and lattice symmetries (Sec. III). We implement field multipole expansion of the eigenmodes of a periodic two-dimensional (2D) photonic structure supporting BICs. We illustrate the developed technique by considering a 2D square array of spheres and extending it to the case of a photonic crystal slab with a 2D array of cylindrical holes (Sec. IV). The developed approach can be easily extended even further to periodic structures with other types of unit-cell and lattice symmetries. The proposed method both provides a deeper understanding of the photonic BIC physics and gives a tool for an effective designing of high- $Q$ resonant photonic systems.

\section{MULTIPOLAR APPROACH}

In this section we consider the modes of a two-dimensional periodic array of dielectric nanoparticles with arbitrary shape (see Fig. 1), and obtain an expression connecting the multipolar content of the field inside and outside the nanoparticles. We denote the VSH as $\mathbf{M}_{p_{r} m n}(k, \mathbf{r})$ (magnetic) and $\mathbf{N}_{p_{r} m n}(k, \mathbf{r})$ (electric), relying on the definition presented in Ref. [73]. We introduce an additional notation $\mathbf{W}_{p_{i} p_{r} m n}$ for both types of VSH, where inversion parity index $p_{i}=(-1)^{n+1}$ for $\mathbf{M}_{p_{r} m n}(k, \mathbf{r})$, and $p_{i}=(-1)^{n}$ for $\mathbf{N}_{p_{r} m n}(k, \mathbf{r})$. Index $n$ is the multipole order, $m$ varies from zero to $n$, and $p_{r}=1$ if $\mathbf{W}_{p_{i} p_{r} m n}$ is even under reflection from the $y=0$ plane $(\varphi \rightarrow-\varphi$ in the spherical system) and $p_{r}=-1$ if it is odd [74].

In a homogeneous medium with permittivity $\varepsilon$, any solution of the Helmholtz equation with wave vector $k=\sqrt{\varepsilon} \frac{\omega}{c}$ can be expanded in terms of electric and magnetic spherical harmonics [75]. The field inside the medium of the $j$ th cell of the array $\mathbf{E}^{\text {in }}$ can be written in terms of multipolar decomposition:

$$
\mathbf{E}^{\mathrm{in}}(\mathbf{r})=\sum_{n=1}^{\infty} \sum_{\substack{m=0 \\ p_{i}, p_{r}}}^{n} E_{0}\left[D_{p_{i} p_{r} m n} \mathbf{W}_{p_{i} p_{r} m n}^{(1)}\left(k_{2}, \mathbf{r}^{\prime}\right) e^{i\left(\mathbf{k}_{b} \cdot \mathbf{r}_{j}\right)}\right],
$$

where $k_{2}=\sqrt{\varepsilon_{2}} \frac{\omega}{c}$ is the wave vector in the material, the superscript (1) stands for spherical Bessel functions in the radial part of the VSH, $\mathbf{k}_{b}$ is the Bloch vector, $\mathbf{r}^{\prime}=\mathbf{r}-\mathbf{r}_{j}$, and $\mathbf{r}_{j}$ is the position of a single sphere (see Fig. 1). $D_{p_{i} p_{r} m n}$

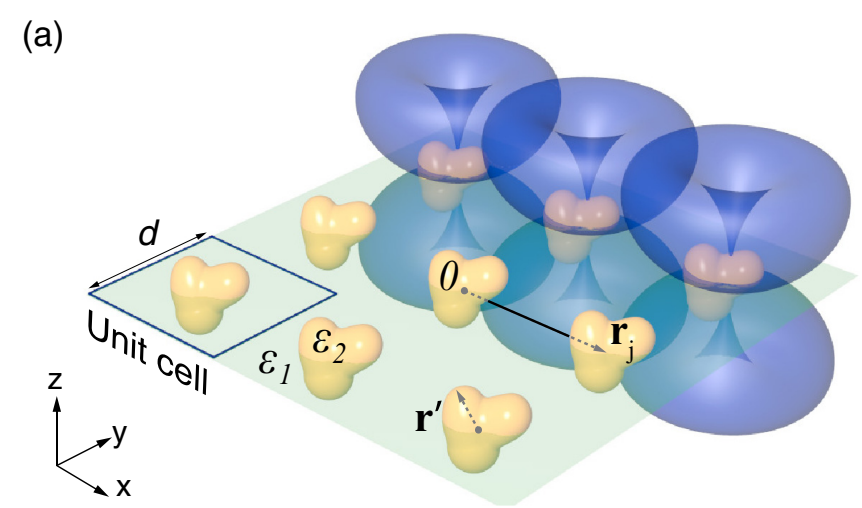

(b) Symmetry-protected BIC:

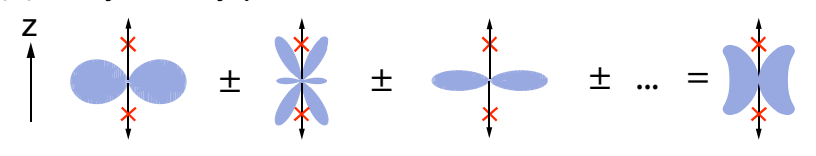

Accidental BIC:

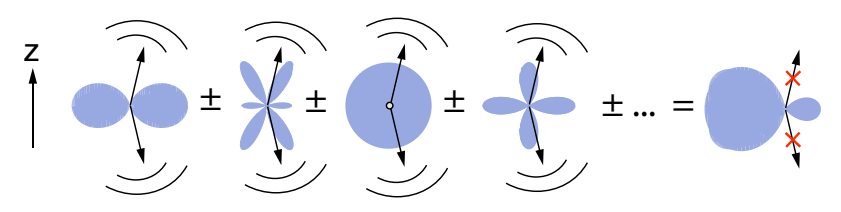

FIG. 1. (a) Metasurface with a square unit cell composed of dielectric meta-atoms. (b) The upper and lower panels show the formation mechanism of symmetry protected $(a t-\Gamma)$ and accidental (off-Г) BIC.

are the coefficients of the multipolar decomposition, which will be discussed in Sec. III. In a similar manner, the field outside the array is expressed as follows (see Appendix B):

$$
\begin{aligned}
\mathbf{E}(\mathbf{r})= & E_{0} \sum_{\mathbf{K}, p_{i}, p_{r}, m, n} \tilde{D}_{p_{i} p_{r} m n} \frac{V_{b} i^{-n}}{2 \pi k_{1}} \iint_{-\infty}^{\infty} d k_{\|} \delta\left(\mathbf{k}_{b}-\mathbf{k}_{\|}-\mathbf{K}\right) \\
& \times \frac{e^{i \mathbf{k r}}}{k_{z}}\left[\mathbf{Y}_{p_{i} p_{r} m n}\left(\frac{\mathbf{k}}{|\mathbf{k}|}\right)\right] .
\end{aligned}
$$

Here $\mathbf{K}$ is the reciprocal-lattice vector, $k_{z}= \pm \sqrt{k_{1}^{2}-k_{\|}^{2}}$, $|\mathbf{k}|=k_{1}=\sqrt{\varepsilon_{1}} \frac{\omega}{c}$, and $V_{b}$ is the volume of the first Brillouin zone. The spherical vector functions $\mathbf{Y}(\mathbf{n})$ depend on the spherical coordinates of a unity vector $\mathbf{n}$ and they are given in Appendix A. Their symmetry coincides with the symmetry of the $\mathbf{W}$ function with identical indices. The relation between $D$ and $\tilde{D}$ is discussed in Appendix C, and for the case of an array of spherical particles coefficients $\tilde{D}$ can be derived analytically.

The summation in Eq. (2) over the reciprocal vectors corresponding to open diffraction channels, i.e., the terms with real $k_{z}$, provides the contribution to the far field. For the frequencies below the diffraction limit, only the zeroth-order term with $\mathbf{K}=0$ gives a nonzero contribution to the far field:

$$
\mathbf{E}(\mathbf{r})=\frac{E_{0} V_{b}}{2 \pi k_{1} k_{1 z}} e^{i \mathbf{k}_{1} \mathbf{r}} \sum_{\substack{p_{i}, p_{r}, m, n}} i^{-n} \tilde{D}_{p_{i} p_{r} m n}\left[\mathbf{Y}_{p_{i} p_{r} m n}\left(\frac{\mathbf{k}_{1}}{k_{1}}\right)\right],
$$


where $\quad \mathbf{k}_{1 \|}=\mathbf{k}_{b}$ and $k_{1 z}= \pm \sqrt{k_{1}^{2}-k_{b}^{2}}$. According to Eq. (3), the contribution of the multipole with numbers $p_{i}, p_{r}, m$, and $n$ to the far field in the direction defined by the wave vector $\mathbf{k}_{1}$ is proportional to the multipole expansion coefficient $\tilde{D}$ and the value of spherical vector function $\mathbf{Y}$ in the given direction. Equation (3) provides the correspondence between the radiation pattern of a single unit cell and the far-field properties of the whole infinite array, allowing for interpreting the BIC in terms of MDM. In strong contrast to a single nanoparticle, where each multipole contributes to the far field, in the case of a subdiffractive array there might be a direction in which none of the multipoles gives any contribution, or alternatively the nonzero contribution of different terms may eventually sum up to zero. The formulated alternative gives a sharp distinction between the symmetry protected and accidental BIC.

\section{A. At- $\Gamma$-point BIC}

The $\Gamma$-point BIC corresponds to the absence of the farfield radiation in the direction along the $z$ axis. Due to the structure of VSH, it appears that a number of multipoles do not radiate in the vertical direction along the $z$ axis. If the field inside a single unit cell consists only of such multipoles, there will be no total radiation in the $z$ direction. This simple fact is illustrated in the upper panel in Fig. 1(b). Noticing that only $\mathbf{Y}_{p_{i} p_{r} \text { 1n }}$ functions with $m=1$ are nonzero in parallel to the $z$-axis direction, we can conclude that at the $\Gamma$ point in the subdiffractive array all the modes which do not contain the harmonics with $m=1$ are symmetry protected BICs. This fundamental conclusion lies at the basis of recent experimental demonstration [10] of lasing with BICs in a 2D subdiffractive array of nanoparticles. The particular operational mode consisted of vertical dipoles oriented along the $z$ axis, thus not contributing to the only open channel. There exists an approach $[1,29]$ in which the eigenmodes at the $\Gamma$ point can radiate in the normal direction $z$ if their fields are odd under $C_{2}^{z}$ rotations and do not have any other rotational symmetry of $C_{n}^{z}$ type. In terms of multipole moments, this follows from the fact that at the $\Gamma$ point any radiative mode should contain multipoles with $m=1$. On the other hand, by virtue of the symmetry, the even modes have zero radiation losses, i.e., an infinite radiation quality factor, and are known as symmetry protected BICs.

\section{B. Off-Г BIC}

Let us now turn to the case of accidental BIC description. In the general case, coefficients $D$ are complex numbers. They define the amplitudes and phase delay between the multipoles. However, in accordance with Ref. [30], if the structure has time reversal and inversion symmetry, the eigenmodes must satisfy the condition $\mathbf{E}(\mathbf{r})=\mathbf{E}^{*}(-\mathbf{r})$. This fact imposes strict conditions on the multipoles' phases; because some of them are even under inversion and some of them are odd, the coefficient $D_{-1 p_{r} m n}$ before the odd ones must be imaginary.

It follows that every term of the sum in Eq. (3) is purely real, and all multipoles are in phase or antiphase. All coefficients depend on $k$-vector and structural parameters and in the case of the off- $\Gamma$ BICs this sum turns to zero. In other words, for the particular $\mathbf{k}_{1}$ all vector harmonics add up to zero in the direction of $\mathbf{k}_{1}$, analogously to the anti-Kerker effect, because they are already in phase and only amplitudes are modulated while the $k$ vector is changed [Fig. 1(b), lower panel]. One can intuitively understand accidental BICs (TM polarized) via a toy dipole model composed of vertical electric and horizontal magnetic dipoles interfering destructively at some $k$ vector [76].

The expansion coefficients $D_{p_{i} p_{r} m n}$ depend on shape of the nanoparticles, material parameters, and symmetry of the lattice. Obviously, the lattice symmetry imposed restrictions on $D_{p_{i} p_{r} m n}$ and some coefficients should vanish due to the symmetry. To explore these selection rules, we employ the group-theoretic approach.

\section{SYMMETRY APPROACH}

The group-theoretic approach is a powerful method which is widely used for analyzing the properties of periodic photonic systems [31,77-80]. In this section, we apply this method to reveal which of the coefficients $D_{p_{i} p_{r} m n}$ are nonzero in accordance with the mode symmetry imposed by the symmetry of the periodic structure $[81,82]$ and to explain the formation of BICs. To make further analysis more illustrative we will provide the example of a square periodic array of dielectric spheres shown in the inset in Fig. 2(a). Nevertheless, it is necessary to highlight that further considerations remain true for any kind of structures with a square lattice with point-group symmetry $D_{4 h}$.

Figure 2(a) shows the dispersion of eigenmodes along the $\overline{\Gamma X}$ direction for a square periodic array of dielectric spheres with permittivity $\varepsilon_{2}=12$ embedded in the air with permittivity $\varepsilon_{1}=1$. The dispersion is calculated numerically using the COMSOL MULTIPHYSICS package. The radius of the spheres is $a=100 \mathrm{~nm}$ and the period of the array is $d=600 \mathrm{~nm}$. Analogous to a dielectric slab waveguide, the eigenmodes of the 2D array are split into transverse electric (TE) and transverse magnetic (TM) modes, which have mainly the $z$ component of magnetic and electric field, correspondingly. Figure 2(b) shows the dependence of the $Q$ factor on the Bloch wave number $k_{x}$ for the modes, which are BICs at the $\Gamma$ point. One can see that, additionally to at- $\Gamma$-BIC, the $\mathrm{TM}_{3}$ mode turns into off-BIC in the middle of the Brillouin band (accidental BIC).

The eigenmodes of periodic structures have certain symmetry in accordance with the fact that every mode is transformed by an irreducible representation of the structure's symmetry group $[83,84]$. While Bloch functions are already the basis functions of the translation group irreducible representation labeled by $\mathbf{k}_{b}$, under point-group operations the basis functions with different $\mathbf{k}_{b}$ transform through each other. They carry high-dimensional representations of the space group. We are interested in the multipolar content of the mode with particular $\mathbf{k}_{b}$, so we look at the point group of $\mathbf{k}_{b}$, i.e., the subgroup of the whole point group, which transforms the $\mathbf{k}_{b}$ into an equivalent. Note that symmetry of the unit cell should be also taken into account since it alters the point group of the full structure. 


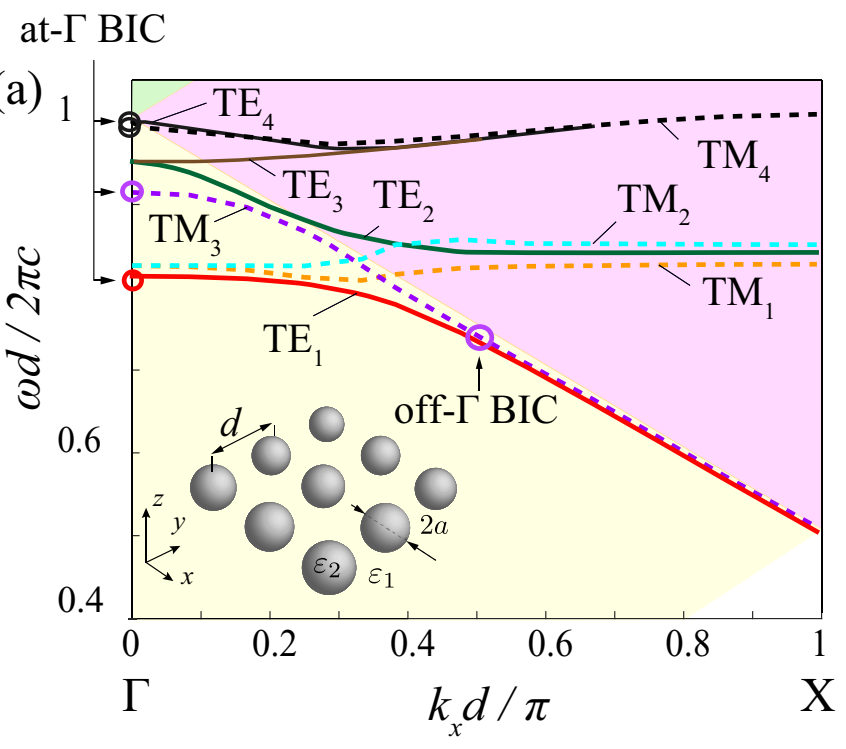

(b)

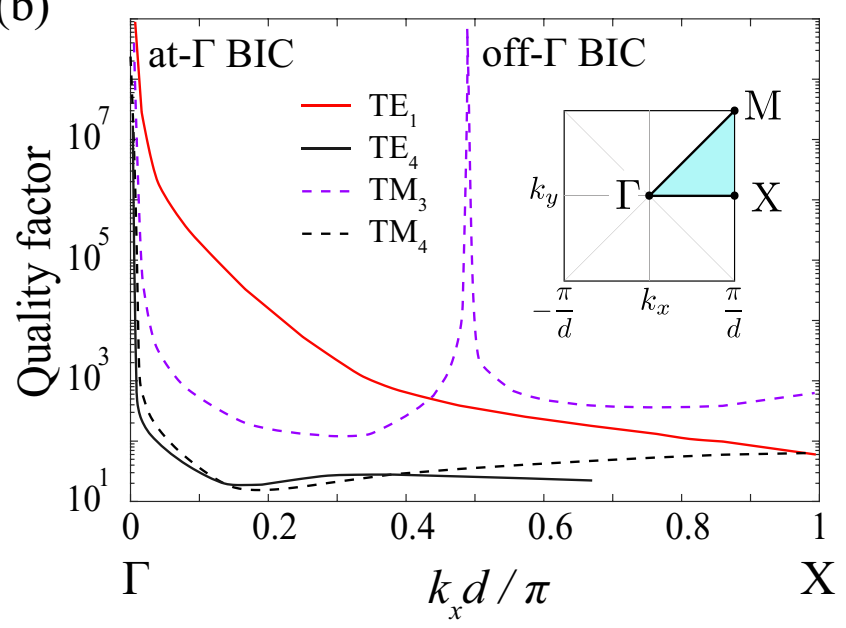

FIG. 2. (a) Band diagram for the square periodic array of dielectric spheres at the $\overline{\Gamma X}$ valley. Period $d=600 \mathrm{~nm}$, nanosphere radius $a=100 \mathrm{~nm}$, dielectric permittivity of the spheres $\varepsilon_{2}=12$, and medium permittivity $\varepsilon_{1}=1$. Modes $\mathrm{TE}_{1}, \mathrm{TE}_{4}, \mathrm{TM}_{3}$, and $\mathrm{TM}_{4}$ are symmetry protected BICs at the $\Gamma$ point. (b) $Q$ factor of the $\mathrm{TM}_{3}$ mode in the $\overline{\Gamma X}$ valley. At the particular wave vector, the $Q$ factor tends to infinity and the accidental BIC appears.

At the $\Gamma$ point, the group of $\mathbf{k}_{b}$ is the whole group $D_{4 h}$, $\mathbf{k}_{b}=0$ and it is not transformed. At $\overline{\Gamma X}$ valleys the point group of $\mathbf{k}_{b}$ is $C_{2 v}$, which consists of $\pi$ rotation around the $x$ or $y$ axis and two plane reflections at $z=0$ and at $y=0$ or $x=0$. These operations keep vector $\mathbf{k}_{b}$ invariant. Analogously, in the $\overline{\Gamma M}$ valley the group is also $C_{2 v}$. Solutions with particular $\mathbf{k}_{b}$ are transformed by one of the $\mathbf{k}_{b}$-group irreducible representations. Thus, since the solution is transformed as a basis function of some particular representation, the multipolar content is strictly limited. Namely, all the multipoles with nonzero contribution must be transformed by an irreducible representation similar to that in the $\mathbf{k}_{b}$ group. We will use common notations for irreducible representations, for example, those listed in Refs. [85,86].

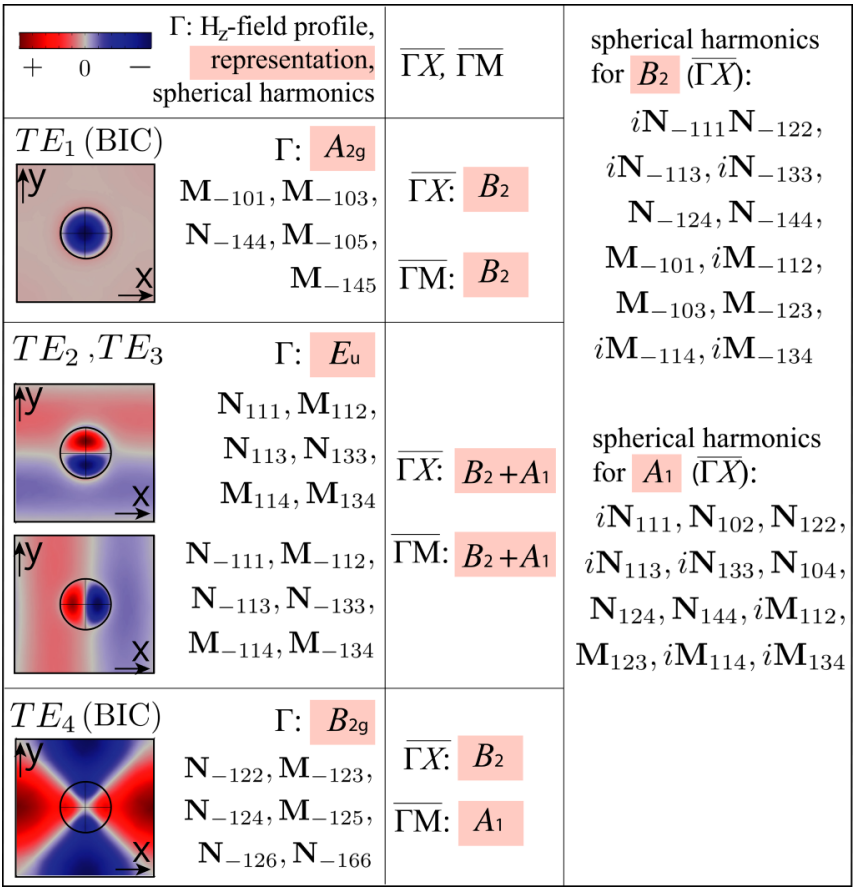

FIG. 3. Multipolar content and irreducible representations of TE modes at the $\Gamma$ point and at $\overline{\Gamma X}$ and $\bar{\Gamma} M$ valleys. Spherical harmonics for $B_{2}$ and $A_{1}$ representations at $\overline{\Gamma X}$ are provided in the right column of the figure. The multipoles for the same representations at $\overline{\Gamma M}$ can be obtained by rotation by $\pi / 4$. The imaginary unit means that coefficients $D$ before these harmonics must be imaginary. The $z$ component of the magnetic field $H_{z}$ is shown in the insets.

\section{A. $\Gamma$ point}

For example, we consider the $\mathrm{TE}_{1}$ mode of the square array, which is transformed by $A_{2 g}$ at the $\Gamma$ point. Under the transformations of the $D_{4 h}$ group the only low-order multipoles transformed by $A_{2 g}$ are magnetic dipole $\mathbf{M}_{-101}$, magnetic octupole $\mathbf{M}_{-103}$, and electric hexadecapole $\mathbf{N}_{-144}$. All of them are invariant under $C_{4}$ rotations, even under inversion and $z=0$-plane reflection, and odd under other $D_{4 h}$ transformations. Higher-order multipoles which behave in the same way are also presented in the multipolar content of this mode.

Analogously, we classify all possible multipoles at the $\Gamma$ point in accordance with their symmetry and provide the multipolar content of the modes (Figs. 3 and 4). Note that $\mathrm{TE}_{2}$ and $\mathrm{TE}_{3}$ modes degenerate, and they transform through each other as two basis functions of the representation $E_{u}$.

\section{B. $\overline{\Gamma X}$ valley}

After the symmetry reduction, when we step out the $\Gamma$ point to the $\overline{\Gamma X}$ valley, some symmetry operations remain, e.g., mirror reflections in $z=0$ and $y=0$ planes and rotation by $\pi$ around the $x$ axis. Eigenmodes must behave in the same way under these symmetry operations, as at the $\Gamma$ point.

As an example, we consider the $\mathrm{TE}_{1}$ mode, which transforms by $A_{2 g}$ at the $\Gamma$ point, and the $\mathrm{TM}_{3}$ mode $\left(A_{1 u}\right)$ at the $\overline{\Gamma X}$ valley. Using the compatibility relations [80], we obtain that the mode which transforms by $A_{2 g}$ at the $\Gamma$ point 


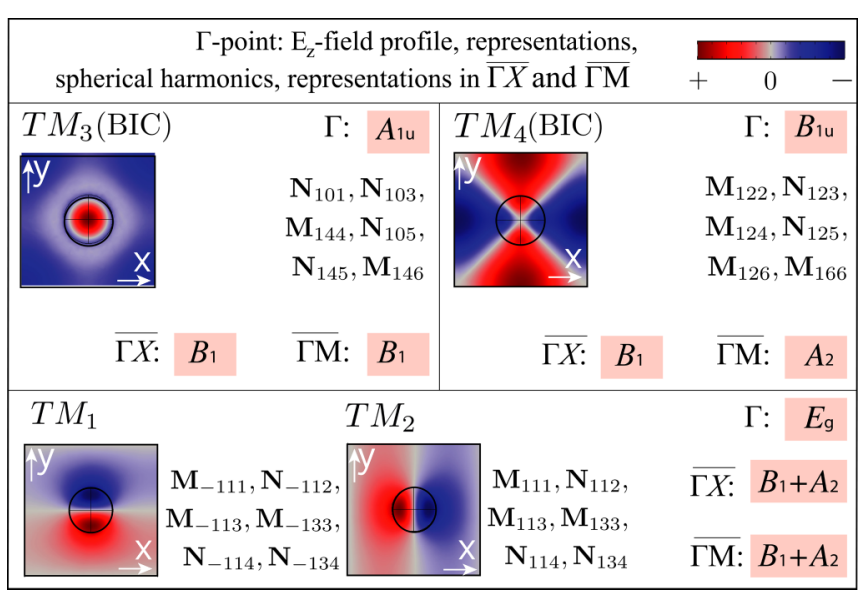

FIG. 4. Multipolar content and irreducible representations of TM modes. The $z$ component of the electric field $E_{z}$ is shown in the insets. Note that representations can be obtained by replacing for TE modes $A_{2} \leftrightarrow A_{1}, B_{2} \leftrightarrow B_{1}$, and $u \leftrightarrow g$, and multipolar content can be obtained by replacing $p_{r} \leftrightarrow-p_{r}$ and $\mathbf{M} \leftrightarrow \mathbf{N}$.

is transformed under $B_{2}$ representation of the group $C_{2 v}$ in the $\overline{\Gamma X}$ valley. The $\mathrm{TM}_{3}$ mode, which is $A_{1 u}$ at the $\Gamma$, is transformed under $B_{1}$ in $\overline{\Gamma X}$. For the $A_{2 g}$ mode at the $\Gamma$ point, which is odd under reflection in the $y=0$ plane and $\pi$ rotation around $x$ and even under reflection in the $z=0$ plane, the only possible multipoles in the $\overline{\Gamma X}$ valley should have the same symmetry properties. For the $A_{1 u}$ mode the possible multipoles in the $\overline{\Gamma X}$ valley must be odd under reflection in the $z=0$ plane and $\pi$ rotation and even under reflection in the $x=0$ plane. Low-order possible multipoles in the $\overline{\Gamma X}$ valley are listed in the right column of Fig. 3 for TE modes, and for TM modes are easily derived by replacing $A_{2} \leftrightarrow$ $A_{1}, B_{2} \leftrightarrow B_{1}, u \leftrightarrow g, p_{r} \leftrightarrow-p_{r}$, and $\mathbf{M} \leftrightarrow \mathbf{N}$. Analogously, for the $\overline{\Gamma M}$ valley we have specific symmetry in the $x=y$ or $x=-y$ direction and possible multipoles are the same as those which are transformed by the same representation in the $\overline{\Gamma X}$ valley, but rotated by $\pi / 4$ with the help of Wigner $D$ matrices $[87,88]$.

\section{MULTIPOLAR COMPOSITION OF THE EIGENMODES IN PERIODIC STRUCTURES}

\section{A. Multipole analysis of metasurfaces and photonic crystal slabs}

Before applying the developed approach to certain structures, we would like to emphasize that the power of the grouptheoretic approach and multipole decomposition method is that these methods can be applied equally for metasurfaces (arrays of meta-atoms) and photonics crystal slabs (slabs with holes). For example, a square array of dielectric spheres and a dielectric slab with circular holes arranged in a square lattice are identical from the point of view of group theory. Therefore, these structures have the same classification of eigenmodes and, more importantly, the same multipole content (Mie resonances). The difference will be only in amplitudes of the multipole coefficients.

At first glance it may seem unphysical to characterize the unit cell with a hole by Mie resonances. This contradiction can be eliminated by means of the proper choice of the unit
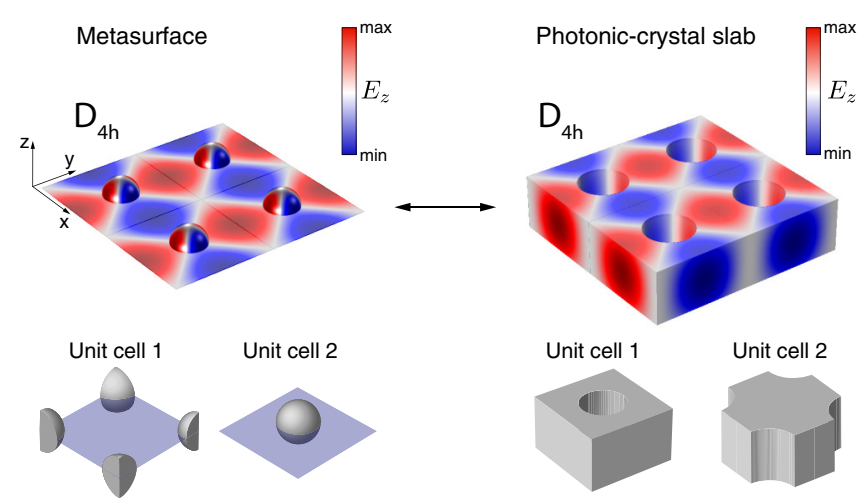

FIG. 5. Distribution of the $E_{z}$ component of the electric field of the modes corresponding to the same irreducible representation $B_{1 u}$ (see Fig. 4) in the photonic crystal slab and metasurface.

cell. Indeed, Fig. 5 shows the modes of the photonic crystal slab and metasurface corresponding to the same irreducible representation $B_{1 u}$ (see Fig. 4). One can see that the mode structures are completely identical. However, it is more physical to assign Mie resonances not to the unit cell with a hole but to the cross-shaped unit cell (see the lower panel in Fig. 5). In particular, it was shown in Ref. [89] that the optical response of both photonic crystal slab and metasurface is dominated by the electric and magnetic Mie-type dipole resonances, and both structures provide $2 \pi$ phase control of light.

\section{B. Periodic arrays of dielectric spheres}

In order to understand how the interference of the multipole moments forms the BICs, we applied a method of multipole decomposition by expanding the eigenmode fields inside the nanoparticles in terms of spherical harmonics $[59,60]$. Figure 6 shows the numerical results for the multipole decomposition for $\mathrm{TE}_{1}, \mathrm{TE}_{4}$, and $\mathrm{TM}_{4}$ modes at the $\Gamma$ point and in a point of the $\Gamma \mathrm{X}$ valley. At the high-symmetry $\Gamma$ point only a small fraction of all possible multipoles is presented, while after lowering the symmetry extra multipoles appear out of the $\Gamma$ point. Figure 7 shows the numerical results for the multipole decomposition for $\mathrm{TM}_{3}$ modes at the $\Gamma$ point, in a point of the $\Gamma \mathrm{X}$ valley, and at the off- $\Gamma$ BIC.

\section{At-Г BIC}

One can see that for the at- $\Gamma \mathrm{TE}_{1}\left(A_{2 g}\right)$ BIC mode the magnetic dipole along the $z$ axis $\left(\mathbf{M}_{-101}\right)$ is mainly contributed [Fig. 6(a)]. Its directivity pattern restricts radiation along the $z$ axis, and the radiation patterns of all remaining multipoles at the $\Gamma$ point are also zero along the $z$ axis. Other directions of radiation are forbidden due to the subdiffraction regime. Similarly, the at- $\Gamma \mathrm{TM}_{3}\left(A_{1 u}\right)$ BIC is formed by the electric dipole along the $z$ axis $\left(\mathbf{N}_{101}\right)$ mostly, prohibiting the radiation in the vertical direction itself [Fig. 7(a)]. Reducing the unit-cell symmetry to $D_{1 h}$ results in the appearance of the in-plane dipole moment and higher multipoles radiating in the vertical direction. Therefore, the BIC turns into a resonant state with a finite $Q$ factor, which could be precisely controlled by the asymmetry degree of the unit cell [90]. The $\mathrm{TE}_{4}\left(B_{2 g}\right)$ and $\mathrm{TM}_{4}\left(B_{1 u}\right)$ modes' lowest multipoles are the 

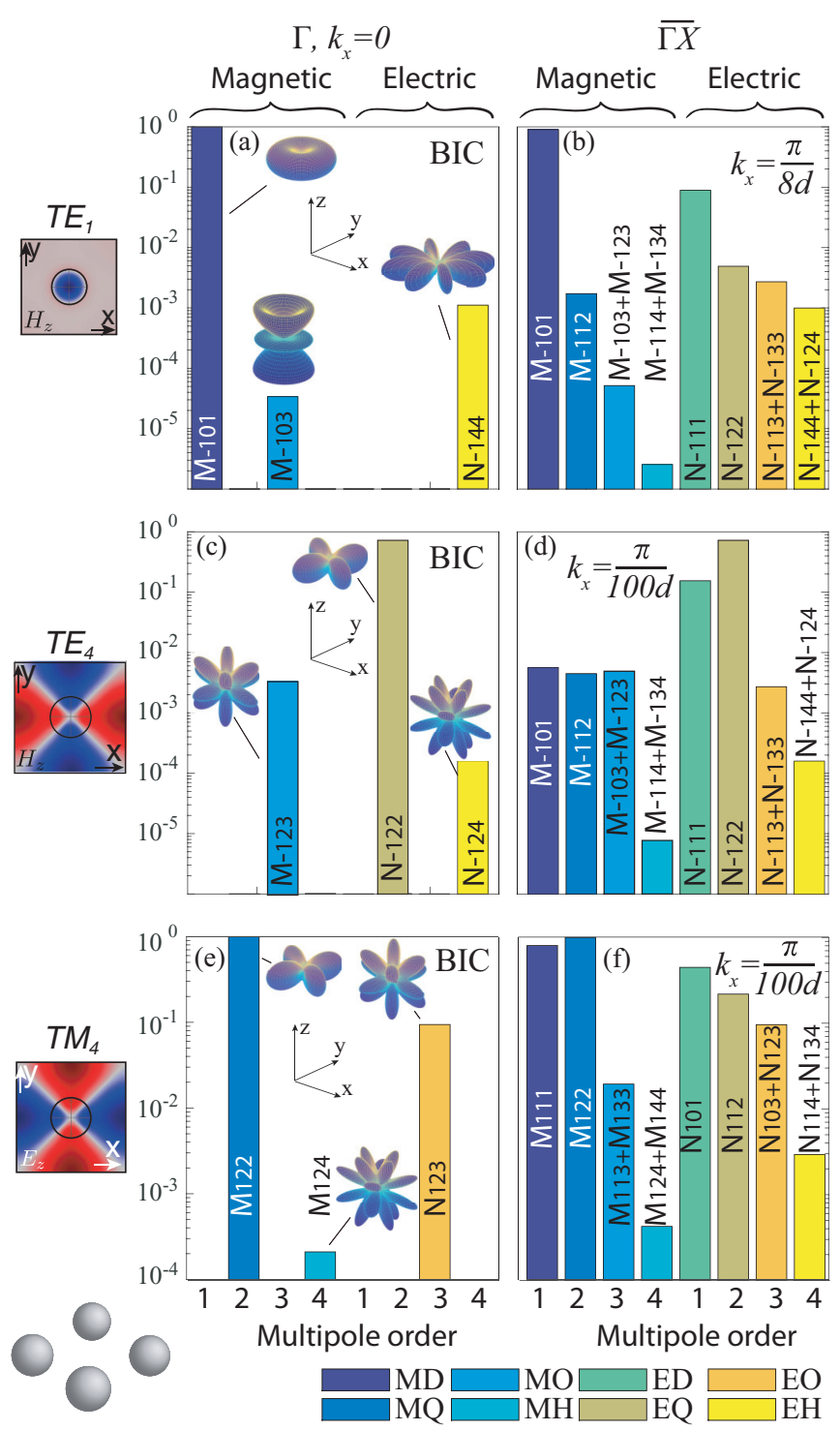

FIG. 6. Multipolar content of $\mathrm{TE}_{1}(\mathrm{a}, \mathrm{b}), \mathrm{TE}_{4}(\mathrm{c}, \mathrm{d})$, and $\mathrm{TM}_{4}$ $(\mathrm{e}, \mathrm{f})$ modes of the metasurface. The vertical axis shows values of normalized coefficients $\tilde{D}$. The horizontal axis shows multipolar order $n$. The letters in the legend encode the multipoles as follows: ED (MD), electric (magnetic) dipole $(n=1)$; EQ (MQ), electric (magnetic) quadrupole $(n=2)$; EO $(\mathrm{MO})$, electric (magnetic) octupole $(n=3)$; $\mathrm{EH}(\mathrm{MH})$, electric (magnetic) hexadecapole $(n=4)$.

electric quadrupole $\mathbf{N}_{-122}$ and the magnetic quadrupole $\mathbf{M}_{122}$, respectively. They have $m=2$, which also prohibits the radiation [Figs. 6(c) and 6(e)]. In contrast to BICs, the radiative modes $\left(E_{g}, E_{u}\right)$ are degenerate at the $\Gamma$ point since they are transformed by the two-dimensional representations. From the symmetry-group approach, we know that $\mathrm{TE}_{2,3}$ and $\mathrm{TM}_{1,2}$ modes contain electric $\mathbf{N}_{ \pm 111}$ and magnetic $\mathbf{M}_{ \pm 111}$ spherical harmonics. The numerical multipole expansion shows that degenerated modes contain in-plane electric or magnetic dipole moments as the main contribution. These numerical results validate the symmetry-group approach for the system with square lattice, confirming that any symmetry protected BIC is characterized by multipole moments with $m \neq 1$.

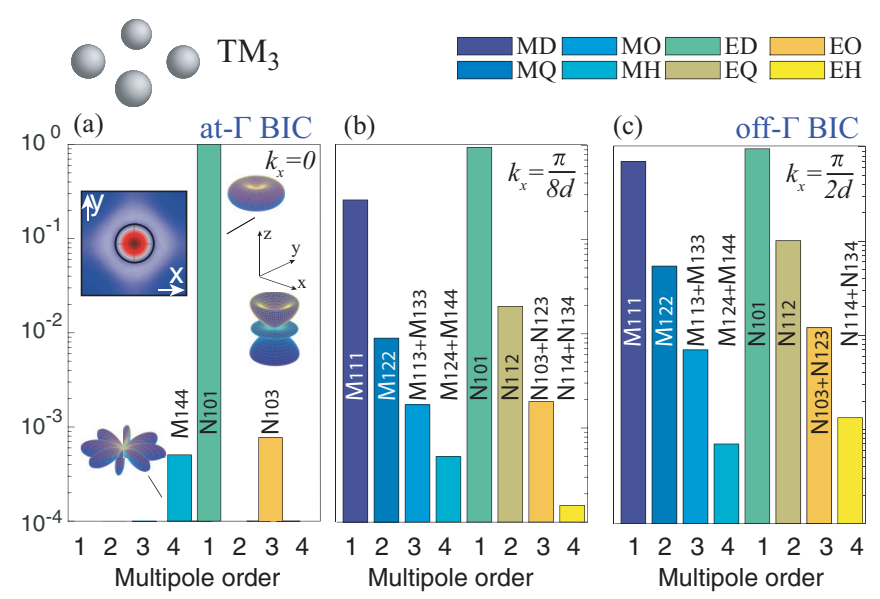

FIG. 7. Multipolar content of the $\mathrm{TM}_{3}$ mode of the metasurface for three $k_{x}$ Bloch vectors corresponding to the symmetry protected BIC (a), leaky mode (b), and accidental BIC (c). The vertical axis shows values of normalized $\tilde{D}$ coefficients; the horizontal axis shows multipolar order $n$. The letters in the legend encode the multipoles as follows: ED (MD), electric (magnetic) dipole $(n=1)$; EQ (MQ), electric (magnetic) quadrupole $(n=2)$; EO (MO), electric (magnetic) octupole $(n=3)$; EH (MH), electric (magnetic) hexadecapole $(n=4)$.

\section{Off-Г BIC}

Away from the $\Gamma$ point other multipoles appear in decomposition [Figs. 6(b), 6(d), 6(f), and 7(b)] and the BIC is destroyed, turning into a resonance state. As mentioned earlier in Sec. II, the accidental off- $\Gamma$ BIC is formed due to either in-phase or antiphase contributions of different multipoles. We obtain the same result for the $\mathrm{TM}_{3}$ mode with off- $\Gamma$ BIC in the $\Gamma X$ valley [Fig. 7(c)]. This mode is transformed by $B_{1}$ representation and consists of the multipoles, which are odd under reflection in the $z=0$ plane and $\pi$ rotation around the $x$ axis and even under $y=0$ reflection, e.g., $i \mathbf{N}_{101}, \mathbf{N}_{112}, i \mathbf{N}_{103}, i \mathbf{N}_{123}, \mathbf{M}_{111}, i \mathbf{M}_{122}, \mathbf{M}_{113}$, and $\mathbf{M}_{133}$. They sum to a TM-polarized wave in the direction given by vector $\mathbf{k}_{1}$ and cancel each other in the off- $\Gamma$ point, forming the accidental BIC, shown in Fig. 2(b). In addition, it is well known that the $z=0$ plane reflection symmetry of the structure is required to obtain the off- $\Gamma$ BIC [30]. Indeed, lacking such symmetry, each mode would contain both odd and even multipoles under reflection in the $z=0$ plane. To restrict the radiation both in the upper and lower half spaces, odd and even multipoles should be summed to zero independently, while for the symmetric structure only one type of multipoles is presented for each mode, which makes it possible to achieve the BIC by tuning the structure parameters.

\section{Photonic crystal slab}

We extend our approach beyond the 2D array of spheres and apply it to the photonic crystal slab of the same symmetry. We consider a dielectric slab with a square array of cylindrical holes studied in Ref. [30]. Both at- $\Gamma$ (symmetry protected) and off- $\Gamma$ (accidental) BICs appear in the lowest TM band, referred to as $\mathrm{TM}_{h}$. This mode has the field profile of the same 

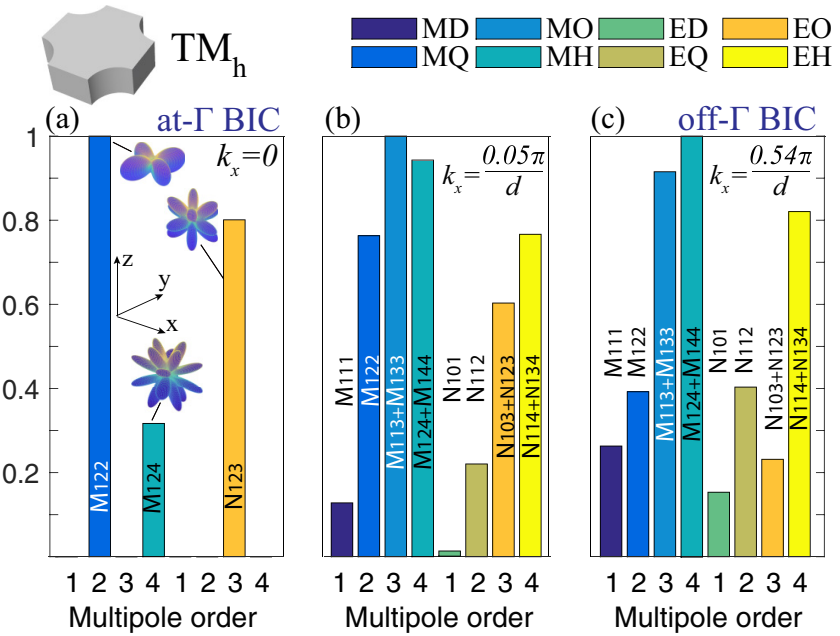

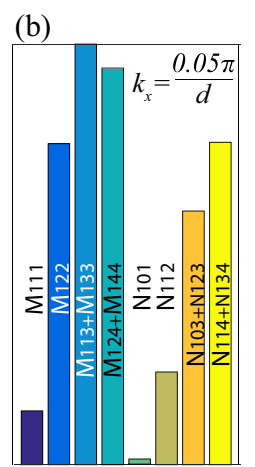

12341234 Multipole order

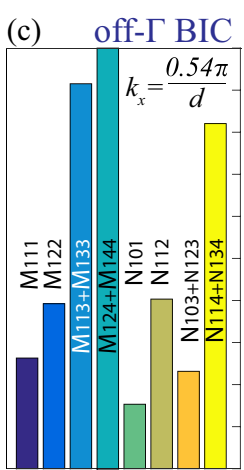

12341234 Multipole order (d)

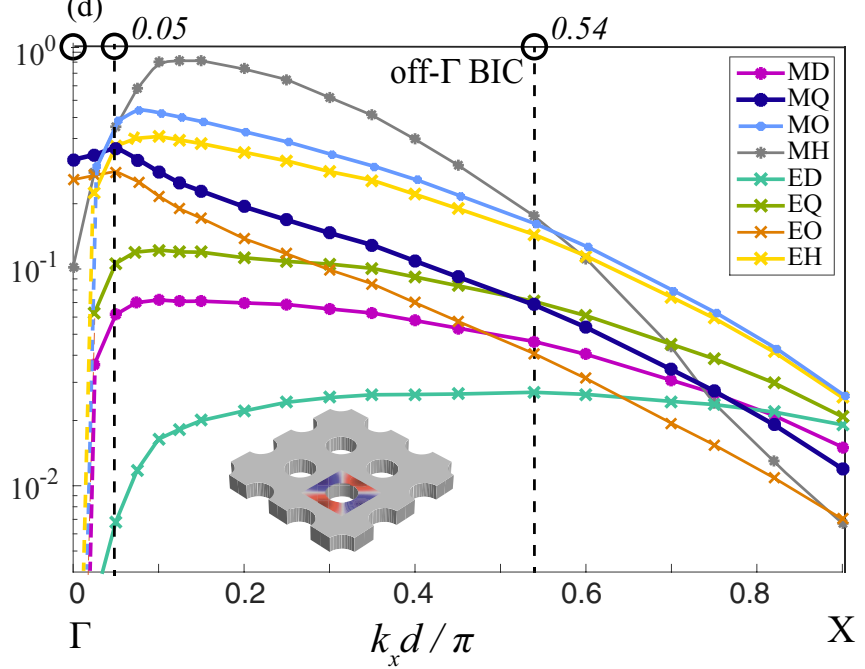

FIG. 8. Multipolar content of the $\mathrm{TM}_{h}$ mode of the $\mathrm{PhC}$ slab for three $k_{x}$ Bloch vectors corresponding to the symmetry protected BIC (a), leaky mode (b), and accidental BIC (c). The vertical axis shows values of normalized $\tilde{D}$ coefficients; the horizontal axis shows multipolar order $n$. Panel (d) depicts how the multipolar contributions change along the $\overline{\Gamma X}$ valley. The letters in the legend encode the multipoles as follows: ED (MD), electric (magnetic) dipole $(n=1)$; EQ (MQ), electric (magnetic) quadrupole $(n=2)$; EO (MO), electric (magnetic) octupole $(n=3)$; EH (MH), electric (magnetic) hexadecapole $(n=4)$.

symmetry as the $\mathrm{TM}_{4}$ mode of the array of the spheres and is transformed by $B_{1 u}$ representation.

The description of the far field defined by Eq. (3) is still valid, but the coefficients $\tilde{D}_{p_{i} p_{r} m n}$ in general cannot be expressed analytically through $D_{p_{i} p_{r} m n}$ (see Appendix C). However, only the multipoles which are presented in the field inside the slab contribute to the far field, $\tilde{D}_{p_{i} p_{r} m n}=0$ if $D_{p_{i} p_{r} m n}=0$. The multipolar content for the considered slab is the same as in the periodic array of spheres because the modes of the slab have the same symmetry as the modes of the array. The multipole decomposition of the $\mathrm{TM}_{h}$ mode [Fig. 8(a)] reveals that a magnetic quadrupole $\mathbf{M}_{122}$ and electric octupole $\mathbf{N}_{123}$ make the major contribution to the at- $\Gamma$ BIC, as well as to the $\mathrm{TM}_{4}$ mode of the array of the spheres. However, the

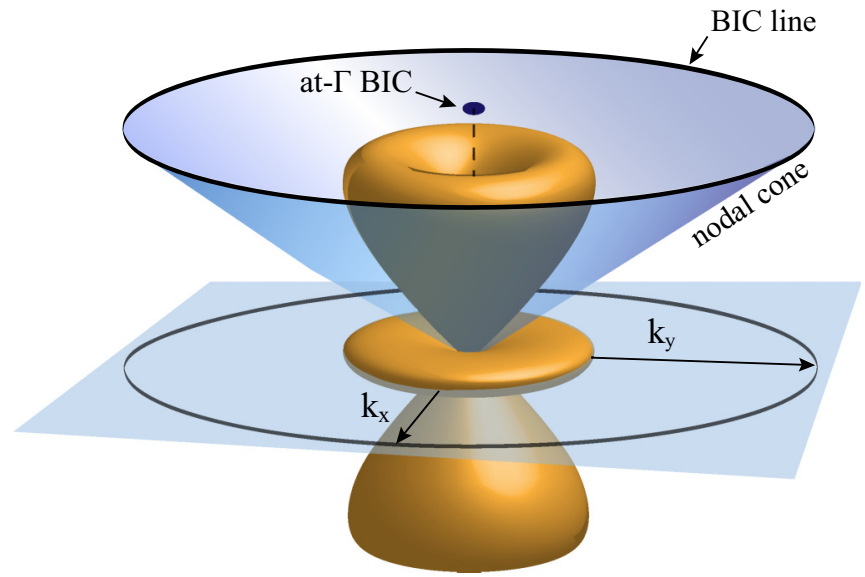

FIG. 9. BIC line formed by the metasurface composed of point octupoles with radiation pattern $\mathbf{N}_{103}$.

$B_{1 u}$ mode of the photonic crystal slab is the lowest-energy TM mode while for the array of spheres it has the highest energy among modes under the diffraction limit. Due to the variational principle [91], for the mode of such symmetry, the electric field is more concentrated inside the high-index material in the case of a photonic crystal slab, minimizing the energy of the mode. Although dispersion curves $\omega(k)$ of the modes $\mathrm{TM}_{4}$ and $\mathrm{TM}_{h}$ behave completely differently, multipole decomposition proves a common origin of them. At the $\Gamma$ point, we obtain contributions of multipoles only with $m=2,6,10$, etc., for both modes, and none of these multipoles contribute in the far field. At the $\overline{\Gamma X}$ valley, the multipolar content of the $\mathrm{TM}_{4}$ and $\mathrm{TM}_{h}$ modes is similar [Figs. 6(e), 6(f), 8(a), and 8(b)]. However, for the $\mathrm{TM}_{h}$ mode it is possible to obtain an accidental off- $\Gamma$ BIC in the $\overline{\Gamma X}$ valley [Fig. 8(c)]. Away from the $\Gamma$ point, multipole contributions change smoothly, keeping the multipolar content invariable, and at a particular wave vector $k_{x}$ multipoles interfere destructively, forming the accidental BIC.

\section{SUMMARY AND OUTLOOK}

Importantly, our approach based on the multipole decomposition analysis of individual meta-atoms not only explains clearly and in simple physical terms the origin of both symmetry protected and accidental bound states in the continuum but also has a prediction power and may be employed for both prediction and engineering of different types of BICs. As an example, we consider a metasurface consisting of meta-atoms packed in a subwavelength 2D lattice, which are polarized purely as octupoles, for example, $\mathbf{M}_{-103}$ (see Fig. 9). Each octupole of this type has a nodal cone and, therefore, we can expect that in a periodic subwavelength array of such meta-atoms BICs form a line in the reciprocal space. However, to observe this phenomenon, the effective polarizability of the unit cell accounting for the interaction between all metaatoms should not depend on the Bloch wave number or have very weak dependence. In other words, observation of BICs in metasurfaces with suppressed spatial dispersion is still a challenging problem. Interestingly, this kind of BIC will be 
observed for the same directions independently of the lattice symmetry of the metasurface.

We can expand this discussion even further, by considering the $\mathbf{M}_{-101}$ dipole. To obtain the $Q$ factor of the at- $\Gamma$ BIC, we should find the dependence of energy-loss rate on the $\mathbf{k}$ vector in Eq. (3), assuming stored energy is almost constant when $\theta_{k} \rightarrow 0$. The asymptotic behavior for $\theta_{k} \rightarrow 0$ of $\mathbf{Y}_{p_{i} p_{r} m n}\left(\frac{\mathbf{k}}{|\mathbf{k}|}\right)$ functions is defined by $\frac{d P_{n}^{m}\left(\cos \theta_{k}\right)}{d \theta_{k}}$. So, $Y_{1-103}\left(\frac{\mathbf{k}}{|\mathbf{k}|}\right) \propto\left(-6 k_{x}+\right.$ $\left.17 k_{x}^{3} / 2\right) \mathbf{e}_{\varphi}$, and $Y_{1-101}\left(\frac{\mathbf{k}}{|\mathbf{k}|}\right) \propto\left(k_{x}-k_{x}^{3} / 6\right) \mathbf{e}_{\varphi}$. We can manage their relative contribution, and at a particular point when the coefficient before the dipole is six times larger we obtain that linear terms cancel each other and the field asymptotic is proportional to $k_{x}^{3}$, so the quality-factor growth is proportional to $k_{x}^{-6}$. A similar effect was observed in Ref. [22] for the photonic crystal slab. However, considering realistic situations, we should take into account all possible multipolar contributions, including terms with $m=1$, where the multipole contribution growth rate plays a role but not the asymptotic behavior. Thus, the multipole origin of the BIC is a query for metasurfaces with a suppressed spatial dispersion.

In summary, we have demonstrated that symmetry protected bound states in the continuum in dielectric metasurfaces and photonic crystal slabs at the frequencies below the diffraction limit are associated with the multipole moments of the elementary meta-atoms which do not radiate in the transverse direction. For any type of metasurfaces, the symmetry protected bound states in the continuum can be observed only if there exist no multipoles with the azimuthal index $m=1$ in the multipole decomposition. The symmetry approach allows us to determine what multipolar content the lattice eigenmodes have, and it can be analogously applied to structures with different symmetries, for example, hexagonal lattices or arrays of nanoparticles of an arbitrary shape and in-plane broken symmetry. Similarly, we have revealed that the accidental bound state in the continuum corresponding to an off- $\Gamma$ point in the reciprocal space is formed due to destructive interference of the multipole fields in the far zone. We have provided general tools for the analysis of bound states in the continuum based on the irreducible representation of the appropriate photonic band. We believe that our results will provide a method for designing high-quality resonant photonic systems based on the physics of bound states in the continuum.

\section{ACKNOWLEDGMENTS}

The authors acknowledge useful discussions with I. D. Avdeev and K. L. Koshelev. This work is supported by the Russian Foundation for Basic Research (Grant No. 1902-00419), the Ministry of Education and Science of the Russian Federation (Grants No. 3.1668.2017/4.6 and No. 3.8891.2017/8.9), and the Grant of the President of the Russian Federation (Grant No. MK-403.2018.2), Russian Foundation for Basic Research 18-02-01206. Y.K. acknowledges support from the Strategic Fund of the Australian National University. Z.S., K.F., M.P., and A.B. acknowledge support from the Foundation for the Advancement of Theoretical Physics and Mathematics "BASIS" (Russia).

Z.S. and K.F. contributed equally to this work.

\section{APPENDIX A: BASIC DEFINITIONS}

Vector spherical harmonics are defined as [73]

$$
\begin{gathered}
\mathbf{M}_{-1 m n}=\nabla \times\left(\mathbf{r} \psi_{-1}^{1} m n\right), \\
\mathbf{N}_{-1}^{1 m n}=\frac{\nabla \times \mathbf{M}_{-1}^{-1} m n}{k},
\end{gathered}
$$

where

$$
\begin{aligned}
& \psi_{1 m n}=\cos m \varphi P_{n}^{m}(\cos \theta) z_{n}(k r), \\
& \psi_{-1 m n}=\sin m \varphi P_{n}^{m}(\cos \theta) z_{n}(k r) .
\end{aligned}
$$

Here $z_{n}(k r)$ can be replaced by a spherical Bessel function of any kind, and $P_{n}^{m}(\cos \theta)$ are the Legendre polynomials. The expansion of the vacuum dyadic Green's function in terms of vector spherical harmonics reads as [92,93]

$$
\begin{aligned}
\hat{\mathbf{G}}_{0}\left(\mathbf{r}, \mathbf{r}^{\prime}\right)= & \frac{i k_{1}}{4 \pi} \sum_{n=1}^{\infty} \sum_{p_{r}= \pm 1} \sum_{m=0}^{n}\left(2-\delta_{0}\right) \frac{2 n+1}{n(n+1)} \frac{(n-m) !}{(n+m) !} \\
& \times\left\{\left[\mathbf{M}_{p_{r} m n}^{(3)}\left(k_{1}, \mathbf{r}\right) \otimes \mathbf{M}_{p_{r} m n}^{(1)}\left(k_{1}, \mathbf{r}^{\prime}\right)\right]\right. \\
& \left.+\left[\mathbf{N}_{p_{r} m n}^{(3)}\left(k_{1}, \mathbf{r}\right) \otimes \mathbf{N}_{p_{r} m n}^{(1)}\left(k_{1}, \mathbf{r}^{\prime}\right)\right]\right\}, \quad \text { for } r>r^{\prime} .
\end{aligned}
$$

Here superscripts (1) and (3) appear, when we replace $z_{n}(\rho)$ by spherical Bessel functions, and the spherical Hankel functions of the first kind are $\delta_{0}=1$ when $m=0$ and $\delta_{0}=0$ when $m \neq 0$.

Spherical vectors $\mathbf{Y}_{p_{i} p_{r} m n}$ denote two types of functions, $\mathbf{X}_{p_{r} m n}$ and $\mathbf{Z}_{p_{r} m n}$, defined as [94]

$$
\begin{aligned}
\mathbf{X}_{-p_{r} m n}\left(\frac{\mathbf{k}}{k}\right) & =\nabla \times\left[\mathbf{k} Y_{p_{r} m n}\left(\frac{\mathbf{k}}{k}\right)\right], \\
\mathbf{Z}_{p_{r} m n}\left(\frac{\mathbf{k}}{k}\right) & =i \frac{\mathbf{k}}{k} \times \mathbf{X}_{-p_{r} m n}\left(\frac{\mathbf{k}}{k}\right),
\end{aligned}
$$

where

$$
\begin{gathered}
Y_{1 m n}(\theta, \varphi)=\cos m \varphi P_{n}^{m}(\cos \theta), \\
Y_{-1 m n}(\theta, \varphi)=\sin m \varphi P_{n}^{m}(\cos \theta) .
\end{gathered}
$$

Here $p_{r}=(-1)^{n+1}$ for $\mathbf{X}$, and $p_{r}=(-1)^{n}$ for $\mathbf{Z}$. Note that the transformation behavior is similar for $\mathbf{W}$ and $\mathbf{Y}, \mathbf{X}$ and $\mathbf{M}$, $\mathbf{Z}$ and $\mathbf{N}$, and $\psi$ and $Y$.

\section{APPENDIX B: LATTICE SUMS OF THE SPHERICAL HARMONICS}

We assume that the multipolar content of the mode is already known, and coefficients in the formula (1) are given. With the help of vacuum dyadic Green's function $\hat{\mathbf{G}}_{0}$, we express the field outside the array:

$$
\begin{aligned}
\mathbf{E}(\mathbf{r}) & =\frac{k_{1}^{2}}{4 \pi} \int d^{3} \mathbf{r}^{\prime \prime} \Delta \varepsilon\left(\mathbf{r}^{\prime \prime}\right) \hat{\mathbf{G}}_{0}\left(\mathbf{r}, \mathbf{r}^{\prime \prime}\right) \mathbf{E}^{\mathrm{in}}\left(\mathbf{r}^{\prime \prime}\right) \\
& =\frac{k_{1}^{2}}{4 \pi}\left(\varepsilon_{2}-\varepsilon_{1}\right) \sum_{j} \int_{V} d^{3} \mathbf{r}^{\prime} \hat{\mathbf{G}}_{0}\left(\mathbf{r}, \mathbf{r}_{j}+\mathbf{r}^{\prime}\right) \mathbf{E}^{\mathrm{in}}\left(\mathbf{r}_{j}+\mathbf{r}^{\prime}\right),
\end{aligned}
$$


where $k_{1}=\sqrt{\varepsilon_{1}} \frac{\omega}{c}$ is the vacuum wave vector, and $V$ is the single nanoparticle's volume.

The Green's function can be also expressed in terms of vector spherical harmonics (see Appendix A). Using the property $\hat{\mathbf{G}}_{0}\left(\mathbf{r}, \mathbf{r}_{j}+\mathbf{r}^{\prime}, \omega\right)=\hat{\mathbf{G}}_{0}\left(\mathbf{r}-\mathbf{r}_{j}, \mathbf{r}^{\prime}, \omega\right)$, and substituting (1) into (B1), we obtain

$$
\begin{aligned}
\mathbf{E}(\mathbf{r})= & E_{0} \sum_{j} \frac{i k_{1}^{3}\left(\varepsilon_{2}-\varepsilon_{1}\right)}{(4 \pi)^{2}} \sum_{p_{i}, p_{r}, n, m} \sum_{p_{p^{\prime}}^{\prime}, p_{r}^{\prime}, n^{\prime}, m^{\prime}}\left(2-\delta_{0}\right) \\
& \times \frac{2 n+1}{n(n+1)} \frac{(n-m) !}{(n+m) !} D_{p_{i}^{\prime} p_{r}^{\prime} n^{\prime} m^{\prime}} \\
& \times \mathbf{W}_{p_{i} p_{r} m n}^{(3)}\left(k_{1}, \mathbf{r}-\mathbf{r}_{j}\right) \cdot e^{i\left(\mathbf{k}_{b} \cdot \mathbf{r}_{j}\right)} \\
& \times \int_{V} d^{3} \mathbf{r}^{\prime}\left[\mathbf{W}_{p_{i} p_{r} m n}^{(1)}\left(k_{1}, \mathbf{r}^{\prime}\right) \cdot \mathbf{W}_{p_{i}^{\prime} p_{r}^{\prime} m^{\prime} n^{\prime}}^{(1)}\left(k_{2}, \mathbf{r}^{\prime}\right)\right] .
\end{aligned}
$$

Here superscript (3) stands for the outgoing spherical Hankel wave. Now we dwell on the case when the array is composed of spherical nanoparticles. Exploiting the VSH orthogonality properties, the integral over the sphere can be taken analytically [75]. It is proportional to Kronecker delta $\delta_{p_{i} p_{i}^{\prime}} \delta_{p_{r} p_{r}^{\prime}} \delta_{m m^{\prime}} \delta_{n n^{\prime}}$ which removes one summation. Combining all coefficients including the integral into $\tilde{D}$ we obtain that the field outside the array is expressed with the formula

$$
\mathbf{E}(\mathbf{r})=E_{0} \sum_{\substack{p_{i}, p_{r}, m, n \\ j}} \tilde{D}_{p_{i} p_{r} m n} \mathbf{W}_{p_{i} p_{r} m n}^{(3)}\left(k_{1}, \mathbf{r}-\mathbf{r}_{j}\right) \cdot e^{i\left(\mathbf{k}_{b} \cdot \mathbf{r}_{j}\right)}
$$

Note that coefficient $\tilde{D}_{p_{i} p_{r} m n}$ is nonzero only if the harmonic with such numbers is presented in the field expansion inside the sphere. If the fields created by each unit cell are already known, we can also start the considerations from this formula.

To obtain formula (2) we exploit the Weyl identity for VSH expansion through the plane waves in the case when $z_{n}(k r)$ is replaced by spherical Hankel functions $[95,96]$ :

$$
\begin{aligned}
& \mathbf{M}_{p_{r} m n}^{(3)}(k, \mathbf{r})=\frac{i^{-n}}{2 \pi k} \iint_{-\infty}^{\infty} d k_{\|} \frac{e^{i\left(k_{x} x+k_{y} y \pm k_{z} z\right)}}{k_{z}}\left[\mathbf{X}_{p_{r} m n}\left(\frac{\mathbf{k}}{k}\right)\right], \\
& \mathbf{N}_{p_{r} m n}^{(3)}(k, \mathbf{r})=\frac{i^{-n}}{2 \pi k} \iint_{-\infty}^{\infty} d k_{\|} \frac{e^{i\left(k_{x} x+k_{y} y \pm k_{z} z\right)}}{k_{z}}\left[\mathbf{Z}_{p_{r} m n}\left(\frac{\mathbf{k}}{k}\right)\right] .
\end{aligned}
$$

The sign before $k_{z}$ depends on the sign of $z$. Redefining the harmonics, we have

$$
\mathbf{W}_{p_{i} p_{r} m n}^{(3)}(k, \mathbf{r})=\frac{i^{-n}}{2 \pi k} \iint_{-\infty}^{\infty} d k_{\|} \frac{e^{i\left(k_{x} x+k_{y} y \pm k_{z} z\right)}}{k_{z}}\left[\mathbf{Y}_{p_{i} p_{r} m n}\left(\frac{\mathbf{k}}{k}\right)\right]
$$

where $k_{z}=\sqrt{k^{2}-k_{x}^{2}-k_{y}^{2}}$. Substituting this expansion into (B3), we get

$$
\begin{aligned}
\mathbf{E}(\mathbf{r})= & E_{0} \sum_{j, p_{i}, p_{r}, n, m} \tilde{D}_{p_{i} p_{r} m n} \cdot e^{i\left(\mathbf{k}_{b} \cdot \mathbf{r}_{j}\right)} \cdot \frac{i^{-n}}{2 \pi k_{1}} \\
& \times \iint_{-\infty}^{\infty} d k_{x} d k_{y} \frac{e^{i\left(\mathbf{k}\left(\mathbf{r}-\mathbf{r}_{j}\right)\right)}}{k_{z}}\left[\mathbf{Y}_{p_{i} p_{r} m n}\left(\frac{\mathbf{k}}{k}\right)\right] .
\end{aligned}
$$

This expansion helps us to apply the summation formula

$$
\sum_{j} e^{i \mathbf{k} \mathbf{x}_{j}}=V_{b} \sum_{\mathbf{K}} \delta(\mathbf{k}-\mathbf{K})
$$

where $\mathbf{K}$ is the reciprocal-lattice vector and $V_{b}$ is the volume of the Brillouin zone $\left(4 \pi^{2} / d^{2}\right.$ for the square lattice), and substituting (B8) into (B7) we obtain Eq. (2).

\section{APPENDIX C: RELATION BETWEEN THE COEFFICIENTS $D$ AND $\tilde{D}$}

The coefficients $D$ in (B2) and $\tilde{D}$ in (B3) are connected by the formula

$$
\begin{aligned}
\tilde{D}_{p_{i} p_{r} m n}= & \frac{i k_{1}^{3}}{(4 \pi)^{2}}\left(2-\delta_{0}\right) \frac{2 n+1}{n(n+1)} \frac{(n-m) !}{(n+m) !} D_{p_{i} p_{r} m n} \\
& \times \sum_{p_{i}^{\prime}, p_{r}^{\prime}, n^{\prime}, m^{\prime}} \int_{V} d^{3} \mathbf{r}^{\prime}\left(\varepsilon_{2}-\varepsilon_{1}\right) \\
& \times\left[\mathbf{W}_{p_{i} p_{r} m n}^{(1)}\left(k_{1}, \mathbf{r}^{\prime}\right) \cdot \mathbf{W}_{p_{i}^{\prime} p_{r}^{\prime} m^{\prime} n^{\prime}}^{(1)}\left(k_{2}, \mathbf{r}^{\prime}\right)\right] .
\end{aligned}
$$

This formula describes both the array of nanoparticles and any photonic crystal slab, but in the case of the array of spheres the integral can be easily taken analytically.

Remark. Here we give the dyadic Green's function $\hat{\mathbf{G}}_{\mathbf{0}}\left(\mathbf{r}, \mathbf{r}^{\prime}\right)$ only for the case when $r>r^{\prime}$. In the case of a photonic crystal slab or nonspherical particles, we also need the part of the Green's function at which $r^{\prime}<r$ [93] to obtain the near field. This will alter the intermediate calculations, since we have to compute the lattice sum for VSH with spherical Bessel functions. Nevertheless, the answer will have the same form.

We apply the orthogonality properties of vector spherical harmonics (see Ref. [75], p. 418), and consider integrals of magnetic and electric harmonics separately. Implementing the angular integration, we reduce the integral to the integral of $r$-dependent Bessel function products, which also can be computed analytically. For magnetic harmonics we have

$$
\begin{aligned}
\tilde{D}_{p_{i} p_{r} m n}^{M}= & i D_{p_{i} p_{r} m n}^{M} a^{2} \varepsilon_{1}\left[k_{1}^{2} j_{n-1}\left(k_{1} a\right) j_{n}\left(k_{2} a\right)\right. \\
& \left.-k_{1} k_{2} j_{n-1}\left(k_{2} a\right) j_{n}\left(k_{1} a\right)\right]
\end{aligned}
$$

where $a$ is the nanoparticle radius, and for electric harmonics we have

$$
\begin{aligned}
\tilde{D}_{p_{i} p_{r} m n}^{N}= & i D_{p_{i} p_{r} m n}^{N} a^{2} \varepsilon_{1}\left\{\frac { n + 1 } { 2 n + 1 } \left[k_{1}^{2} j_{n-2}\left(k_{1} a\right) j_{n-1}\left(k_{2} a\right)\right.\right. \\
& \left.-k_{1} k_{2} j_{n-2}\left(k_{2} a\right) j_{n-1}\left(k_{1} a\right)\right] \\
& +\frac{n}{2 n+1}\left[k_{1}^{2} j_{n}\left(k_{1} a\right) j_{n+1}\left(k_{2} a\right)\right. \\
& \left.\left.-k_{1} k_{2} j_{n}\left(k_{2} a\right) j_{n+1}\left(k_{1} a\right)\right]\right\}
\end{aligned}
$$

Note that this expression turns to zero at some frequencies, so we can have zero $\tilde{D}$ when $D$ is nonzero. This refers to the anapoles of the spherical nanoparticles. The frequency where the anapole appears is the same as for the single isolated nanoparticle. 
If we have another type of surface, for example, a photonic crystal slab with holes or an array of cylinders, the orthogonality property cannot be applied and the integral $\int_{V} d^{3} \mathbf{r}^{\prime} \Delta \varepsilon\left[\mathbf{W}_{p_{i} p_{r} m n}^{(1)}\left(k_{1}, \mathbf{r}^{\prime}\right) \cdot \mathbf{W}_{p_{i}^{\prime} p_{r}^{\prime} m^{\prime} n^{\prime}}^{(1)}\left(k_{2}, \mathbf{r}^{\prime}\right)\right]$ will mix some harmonics. However, all the harmonics, which admix, are already presented in the expansion of the field inside the cell. This will just alter the coefficients before the outgoing multipoles, but not the multipolar content.
[1] C. W. Hsu, B. Zhen, A. D. Stone, J. D. Joannopoulos, and M. Soljačić, Bound states in the continuum, Nat. Rev. Mater. 1, 16048 (2016).

[2] K. Koshelev, A. Bogdanov, and Y. Kivshar, Meta-optics and bound states in the continuum, Sci. Bull. 64, 836 (2018).

[3] K. Koshelev, G. Favraud, A. Bogdanov, Y. Kivshar, and A. Fratalocchi, Nonradiating photonics with resonant dielectric nanostructures, Nanophotonics 8, 725 (2019).

[4] J. V. Neumann and E. P. Wigner, Uber merkwurdige diskrete Eigenwerte, Phys. Z. 30, 465 (1929).

[5] P. Paddon and J. F. Young, Two-dimensional vector-coupledmode theory for textured planar waveguides, Phys. Rev. B 61, 2090 (2000).

[6] E. N. Bulgakov and A. F. Sadreev, Bound states in the continuum in photonic waveguides inspired by defects, Phys. Rev. B 78, 075105 (2008).

[7] D. C. Marinica, A. G. Borisov, and S. V. Shabanov, Bound States in the Continuum in Photonics, Phys. Rev. Lett. 100, 183902 (2008).

[8] F. Dreisow, A. Szameit, M. Heinrich, R. Keil, S. Nolte, A. Tünnermann, and S. Longhi, Adiabatic transfer of light via a continuum in optical waveguides, Opt. Lett. 34, 2405 (2009).

[9] C. M. Gentry and M. A. Popović, Dark state lasers, Opt. Lett. 39, 4136 (2014).

[10] S. T. Ha, Y. H. Fu, N. K. Emani, Z. Pan, R. M. Bakker, R. Paniagua-Dominguez, and A. I. Kuznetsov, Directional lasing in resonant semiconductor nanoantenna arrays, Nat. Nanotechnol. 13, 1042 (2018).

[11] A. Kodigala, T. Lepetit, Q. Gu, B. Bahari, Y. Fainman, and B. Kanté, Lasing action from photonic bound states in continuum, Nature (London) 541, 196 (2017).

[12] E. Penzo, S. Romano, Y. Wang, S. Dhuey, L. Dal Negro, V. Mocella, and S. Cabrini, Patterning of electrically tunable lightemitting photonic structures demonstrating bound states in the continuum, J. Vac. Sci. Technol. B 35, 06G401 (2017).

[13] M. Rybin and Y. Kivshar, Optical physics: Supercavity lasing, Nature (London) 541, 164 (2017).

[14] J. M. Foley and J. D. Phillips, Normal incidence narrowband transmission filtering capabilities using symmetry protected modes of a subwavelength, dielectric grating, Opt. Lett. 40, 2637 (2015).

[15] J. M. Foley, S. M. Young, and J. D. Phillips, Symmetryprotected mode coupling near normal incidence for narrowband transmission filtering in a dielectric grating, Phys. Rev. B 89, 165111 (2014).

[16] S. Romano, A. Lamberti, M. Masullo, E. Penzo, S. Cabrini, I. Rendina, and V. Mocella, Optical biosensors based on photonic crystals supporting bound states in the continuum, Materials 11, 526 (2018).

[17] S. Romano, G. Zito, S. Torino, G. Calafiore, E. Penzo, G. Coppola, S. Cabrini, I. Rendina, and V. Mocella, Labelfree sensing of ultralow-weight molecules with all-dielectric metasurfaces supporting bound states in the continuum, Photon. Res. 6, 726 (2018).

[18] L. Carletti, K. Koshelev, C. De Angelis, and Y. Kivshar, Giant Nonlinear Response at the Nanoscale Driven by Bound States in the Continuum, Phys. Rev. Lett. 121, 033903 (2018).

[19] X. Gao, C. W. Hsu, B. Zhen, X. Lin, J. D. Joannopoulos, M. Soljačić, and H. Chen, Formation mechanism of guided resonances and bound states in the continuum in photonic crystal slabs, Sci. Rep. 6, 31908 (2016).

[20] E. N. Bulgakov and A. F. Sadreev, Robust bound state in the continuum in a nonlinear microcavity embedded in a photonic crystal waveguide, Opt. Lett. 39, 5212 (2014).

[21] S. I. Azzam, V. M. Shalaev, A. Boltasseva, and A. V. Kildishev, Formation of Bound States in the Continuum in Hybrid Plasmonic-Photonic Systems, Phys. Rev. Lett. 121, 253901 (2018).

[22] J. Jin, X. Yin, L. Ni, M. Soljačić, B. Zhen, and C. Peng, Topologically enabled ultra-high-q guided resonances robust to out-of-plane scattering, arXiv:1812.00892.

[23] W. Chen, Y. Chen, and W. Liu, Singularities and Poincaré Indices of Electromagnetic Multipoles, Phys. Rev. Lett. 122, 153907 (2019).

[24] D. R. Abujetas, A. Barreda, F. Moreno, J. J. Saenz, A. Litman, J.-M. Geffrin, and J. A. Sanchez-Gil, Brewster quasi bound states in the continuum in all-dielectric metasurfaces from single magnetic-dipole resonance meta-atoms, arXiv:1902.07148.

[25] Y. V. Kartashov, C. Milián, V. V. Konotop, and L. Torner, Bound states in the continuum in a two-dimensional PT-symmetric system, Opt. Lett. 43, 575 (2018).

[26] S. Romano, G. Zito, S. Managò, G. Calafiore, E. Penzo, S. Cabrini, A. C. De Luca, and V. Mocella, Surface-enhanced raman and fluorescence spectroscopy with an all-dielectric metasurface, J. Phys. Chem. C 122, 19738 (2018).

[27] E. Bulgakov and A. Sadreev, Trapping of light with angular orbital momentum above the light cone, Advanced Electromagnetics 6, 1 (2017).

[28] C. Wei Hsu, B. Zhen, S.-L. Chua, S. G. Johnson, J. D. Joannopoulos, and M. Soljačić, Bloch surface eigenstates within the radiation continuum, Light Sci. Appl. 2, e84 (2013).

[29] B. Zhen, C. W. Hsu, L. Lu, A. D. Stone, and M. Soljačić, Topological Nature of Optical Bound States in the Continuum, Phys. Rev. Lett. 113, 257401 (2014).

[30] C. W. Hsu, B. Zhen, J. Lee, S.-L. Chua, S. G. Johnson, J. D. Joannopoulos, and M. Soljačić, Observation of trapped light within the radiation continuum, Nature (London) 499, 188 (2013).

[31] P. Yu, A. S. Kupriianov, V. Dmitriev, and V. R. Tuz, Alldielectric metasurfaces with trapped modes: Group-theoretical description, J. Appl. Phys. 125, 143101 (2019).

[32] L. Ni, Z. Wang, C. Peng, and Z. Li, Tunable optical bound states in the continuum beyond in-plane symmetry protection, Phys. Rev. B 94, 245148 (2016). 
[33] H. Friedrich and D. Wintgen, Interfering resonances and bound states in the continuum, Phys. Rev. A 32, 3231 (1985).

[34] L. C. Andreani and D. Gerace, Photonic-crystal slabs with a triangular lattice of triangular holes investigated using a guidedmode expansion method, Phys. Rev. B 73, 235114 (2006).

[35] E. A. Muljarov, W. Langbein, and R. Zimmermann, BrillouinWigner perturbation theory in open electromagnetic systems, Europhys. Lett. 92, 50010 (2010).

[36] T. Weiss and E. A. Muljarov, How to calculate the pole expansion of the optical scattering matrix from the resonant states, Phys. Rev. B 98, 085433 (2018).

[37] C. Blanchard, P. Viktorovitch, and X. Letartre, Perturbation approach for the control of the quality factor in photonic crystal membranes: Application to selective absorbers, Phys. Rev. A 90, 033824 (2014).

[38] A.-L. Fehrembach and A. Sentenac, Study of waveguide grating eigenmodes for unpolarized filtering applications, JOSA A $\mathbf{2 0}$, 481 (2003).

[39] M. G. Moharam and T. K. Gaylord, Rigorous coupled-wave analysis of planar-grating diffraction, JOSA 71, 811 (1981).

[40] S. G. Tikhodeev, A. L. Yablonskii, E. A. Muljarov, N. A. Gippius, and T. Ishihara, Quasiguided modes and optical properties of photonic crystal slabs, Phys. Rev. B 66, 045102 (2002).

[41] V. Liu and S. Fan, S4: A free electromagnetic solver for layered periodic structures, Comput. Phys. Commun. 183, 2233 (2012).

[42] B. Vial, F. Zolla, A. Nicolet, and M. Commandré, Quasimodal expansion of electromagnetic fields in open two-dimensional structures, Phys. Rev. A 89, 023829 (2014).

[43] P. Lalanne, W. Yan, K. Vynck, C. Sauvan, and J.-P. Hugonin, Light interaction with photonic and plasmonic resonances, Laser Photonics Rev. 12, 1700113 (2018).

[44] P. Lalanne, J. P. Hugonin, and P. Chavel, Optical properties of deep lamellar gratings: A coupled bloch-mode insight, J. Lightwave Technol. 24, 2442 (2006).

[45] J. M. Pottage, E. Silvestre, and P. S. J. Russell, Vertical-cavity surface-emitting resonances in photonic crystal films, JOSA A 18, 442 (2001).

[46] A. Modinos, N. Stefanou, and V. Yannopapas, Applications of the layer-kkr method to photonic crystals, Opt. Express 8, 197 (2001).

[47] A. F. Sadreev, Feshbach projection formalism for transmission through a time-periodic potential, Phys. Rev. E 86, 056211 (2012).

[48] J. S. T. Gongora, G. Favraud, and A. Fratalocchi, Fundamental and high-order anapoles in all-dielectric metamaterials via Fano-Feshbach modes competition, Nanotechnology 28, 104001 (2017).

[49] D. Felbacq and E. Rousseau, All-optical photonic band control in a quantum metamaterial, Ann. Phys. (NY) 529, 1600371 (2017).

[50] R. F. Ndangali and S. V. Shabanov, Electromagnetic bound states in the radiation continuum for periodic double arrays of subwavelength dielectric cylinders, J. Math. Phys. 51(10), 102901 (2010).

[51] Y. Yang, C. Peng, Y. Liang, Z. Li, and S. Noda, Analytical Perspective for Bound States in the Continuum in Photonic Crystal Slabs, Phys. Rev. Lett. 113, 037401 (2014).

[52] E. N. Bulgakov and A. F. Sadreev, Bloch bound states in the radiation continuum in a periodic array of dielectric rods, Phys. Rev. A 90, 053801 (2014).
[53] C. Blanchard, J.-P. Hugonin, and C. Sauvan, Fano resonances in photonic crystal slabs near optical bound states in the continuum, Phys. Rev. B 94, 155303 (2016).

[54] L. Yuan and Y. Y. Lu, Bound states in the continuum on periodic structures: Perturbation theory and robustness, Opt. Lett. 42, 4490 (2017).

[55] E. N. Bulgakov, A. F. Sadreev, and D. N. Maksimov, Light trapping above the light cone in one-dimensional arrays of dielectric spheres, Applied Sciences 7, 147 (2017).

[56] K. L. Koshelev, S. K. Sychev, Z. F. Sadrieva, A. A. Bogdanov, and I. V. Iorsh, Strong coupling between excitons in transition metal dichalcogenides and optical bound states in the continuum, Phys. Rev. B 98, 161113(R) (2018).

[57] A. I. Ovcharenko, C. Blanchard, J.-P. Hugonin, and C. Sauvan, Bound states in the continuum in symmetric and asymmetric photonic crystal slabs, arXiv:1907.09330.

[58] Z. F. Sadrieva, M. A. Belyakov, M. A. Balezin, P. V. Kapitanova, E. A. Nenasheva, A. F. Sadreev, and A. A. Bogdanov, Experimental observation of a symmetry-protected bound state in the continuum in a chain of dielectric disks, Phys. Rev. A 99, 053804 (2019).

[59] J. Jackson, Classical Electrodynamics (Wiley, New York, 1999).

[60] P. Grahn, A. Shevchenko, and M. Kaivola, Electromagnetic multipole theory for optical nanomaterials, New J. Phys. 14, 093033 (2012).

[61] S. S. Kruk, R. Camacho-Morales, L. Xu, M. Rahmani, D. Smirnova, L. Wang, H. H. Tan, C. Jagadish, D. N. Neshev, and Y. S. Kivshar, Nonlinear optical magnetism revealed by second-harmonic generation in nanoantennas, Nano Lett. 17, 3914 (2017).

[62] A. B. Evlyukhin, C. Reinhardt, A. Seidel, B. S. Luk'yanchuk, and B. N. Chichkov, Optical response features of Sinanoparticle arrays, Phys. Rev. B 82, 045404 (2010).

[63] R. Paniagua-Domínguez, Y. F. Yu, A. E. Miroshnichenko, L. A. Krivitsky, Y. H. Fu, V. Valuckas, L. Gonzaga, Y. T. Toh, A. Y. S. Kay, B. Luk'yanchuk, and A. I. Kuznetsov, Generalized Brewster effect in dielectric metasurfaces, Nat. Commun. 7, 10362 (2016).

[64] T. Lepetit and B. Kanté, Controlling multipolar radiation with symmetries for electromagnetic bound states in the continuum, Phys. Rev. B 90, 241103(R) (2014).

[65] A. I. Kuznetsov, A. E. Miroshnichenko, M. L. Brongersma, Y. S. Kivshar, and B. Luk'yanchuk, Optically resonant dielectric nanostructures, Science 354, aag2472 (2016).

[66] M. Panmai, J. Xiang, Z. Sun, Y. Peng, H. Liu, H. Liu, Q. Dai, S. Tie, and S. Lan, All-silicon-based nano-antennas for wavelength and polarization demultiplexing, Opt. Express 26, 12344 (2018).

[67] K. Baryshnikova, D. Filonov, C. Simovski, A. Evlyukhin, A. Kadochkin, E. Nenasheva, P. Ginzburg, and A. S. Shalin, Giant magnetoelectric field separation via anapole-type states in high-index dielectric structures, Phys. Rev. B 98, 165419 (2018).

[68] P. C. Wu, C. Y. Liao, V. Savinov, T. L. Chung, W. Chen, Y.-W. Huang, P. R. Wu, Y.-H. Chen, A.-Q. Liu, N. Zheludev, and D. P. Tsai, Optical anapole metamaterial, ACS Nano 12, 1920 (2018).

[69] W. Hsu, Novel trapping and scattering of light in resonant nanophotonic structures, Doctoral dissertation, Harvard University, Graduate School of Arts \& Sciences, Ph.D. thesis, 2015. 
[70] A. V. Poshakinskiy and A. N. Poddubny, Optomechanical Kerker Effect, Phys. Rev. X 9, 011008 (2019).

[71] H. K. Shamkhi, K. V. Baryshnikova, A. Sayanskiy, P. Kapitanova, P. D. Terekhov, P. Belov, A. Karabchevsky, A. B. Evlyukhin, Y. Kivshar, and A. S. Shalin, Transverse Scattering and Generalized Kerker Effects in All-Dielectric Mie-Resonant Metaoptics, Phys. Rev. Lett. 122, 193905 (2019).

[72] W. Liu and Y. S. Kivshar, Generalized kerker effects in nanophotonics and meta-optics (invited), Opt. Express 26, 13085 (2018).

[73] C. F. Bohren and D. R. Huffman, Absorption and Scattering of Light by Small Particles (Wiley, New York, 1983), pp. 83-84.

[74] K. Frizyuk, I. Volkovskaya, D. Smirnova, A. Poddubny, and M. Petrov, Second-harmonic generation in mie-resonant dielectric nanoparticles made of noncentrosymmetric materials, Phys. Rev. B 99, 075425 (2019).

[75] J. A. Stratton, Electromagnetic Theory (Wiley - Interscience, A John Wiley \& Sons. Inc., Hoboken, New Jersey, 2007), pp. 392-423.

[76] H. M. Doeleman, F. Monticone, W. den Hollander, A. Alù, and A. F. Koenderink, Experimental observation of a polarization vortex at an optical bound state in the continuum, Nat. Photon. 12, 397 (2018).

[77] W. Hergert and M. Dane, Group theoretical investigations of photonic band structures, Phys. Status Solidi A 197, 620 (2003).

[78] K. Ohtaka and Y. Tanabe, Photonic band using vector spherical waves. I. Various properties of Bloch electric fields and heavy photons, J. Phys. Soc. Jpn. 65, 2265 (1996).

[79] K. Ohtaka and Y. Tanabe, Photonic bands using vector spherical waves. III. Group-theoretical treatment, J. Phys. Soc. Jpn. 65, 2670 (1996).

[80] K. Sakoda, Optical Properties of Photonic Crystals, Springer Series in Optical Sciences Vol. 80 (Springer, New York, 2001).

[81] S. Hayami, M. Yatsushiro, Y. Yanagi, and H. Kusunose, Classification of atomic-scale multipoles under crystallographic point groups and application to linear response tensors, Phys. Rev. B 98, 165110 (2018).

[82] A. Gelessus, W. Thiel, and W. Weber, Multipoles and symmetry, J. Chem. Educ. 72, 505 (1995).

[83] V. M. Agranovich and V. Ginzburg, Crystal Optics with Spatial Dispersion, and Excitons, Springer Series in Solid-State Science, Vol. 42 (Springer, New York, 2013).
[84] E. Ivchenko and G. Pikus, Crystal symmetry, in Superlattices and Other Heterostructures, Springer Series in Solid-State Sciences Vol. 110 (Springer-Verlag, Berlin, 1995).

[85] Point group symmetry, https://www.staff.ncl.ac.uk/j.p.goss/ symmetry/index.html.

[86] Character tables for point groups used in chemistry, http:// gernot-katzers-spice-pages.com/character_tables/index.html.

[87] Z. Huayong and H. Yiping, Addition theorem for the spherical vector wave functions and its application to the beam shape coefficients, J. Opt. Soc. Am. B 25, 255 (2008).

[88] G. Aubert, An alternative to Wigner d-matrices for rotating real spherical harmonics, AIP Advances 3, 062121 (2013).

[89] Q. Yang, S. Kruk, Y. K. Srivastava, K. Koshelev, R. Singh, J. Han, Y. Kivshar, and I. Shadrivov, Dielectric membrane Mieresonant metasurfaces, in Conference on Lasers and ElectroOptics, OSA Technical Digest (Optical Society of America, 2019), paper SW3F.5.

[90] K. Koshelev, S. Lepeshov, M. Liu, A. Bogdanov, and Y. Kivshar, Asymmetric Metasurfaces with High- $q$ Resonances Governed by Bound States in the Continuum, Phys. Rev. Lett. 121, 193903 (2018).

[91] J. D. Joannopoulos, S. G. Johnson, J. N. Winn, and R. D. Meade, Photonic Crystals: Molding the Flow of Light, 2nd ed. (Princeton University, Princeton, NJ, 2008).

[92] C. T. Tai and C. Yeh, Dyadic Green's Functions in Electromagnetic Theory, IEEE Antennas and Propagation Group Newsletter, 2nd ed. (IEEE, New York, 1994).

[93] L.-W. Li, P.-S. Kooi, M.-S. Leong, and T.-S. Yee, Electromagnetic dyadic Green's function in spherically multilayered media, IEEE Trans. Microwave Theory Tech. 42, 2302 (1994).

[94] R. Alaee, C. Rockstuhl, and I. Fernandez-Corbaton, Exact multipolar decompositions with applications in nanophotonics, Adv. Opt. Mater 7, 1800783 (2019).

[95] R. C. Wittmann, Spherical wave operators and the translation formulas, IEEE Trans. Antennas Propag. 36, 1078 (1988).

[96] B. Stout, Spherical harmonic lattice sums for gratings, in Gratings: Theory and Numeric Applications, edited by E. Popov (Universite d'Aix-Marseille, Marseille, France, 2012), Vol. 6. 